\title{
The Model and Application of Heat and Mass Transfer of Chinese Cuisine
}

ISSN: 2640-9208

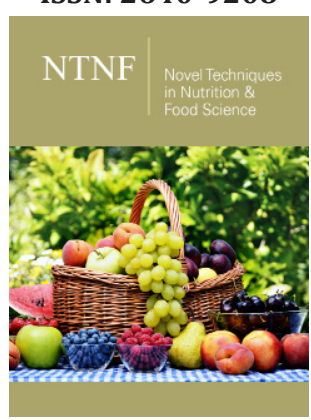

*Corresponding author: Wang Yong, Hangzhou Robam Appliances CO., LTD. Hangzhou, China

Submission: 非 April 07, 2021

Published: 監May 6, 2021

Volume 5 - Issue 5

How to cite this article: Wang Yong, Jiang Xin. The Model and Application of Heat and Mass Transfer of Chinese Cuisine. Nov Tech Nutri Food Sci. 5(5). NTNF. 000625. 2021 DOI: 10.31031/NTNF.2021.05.000625

Copyright@ Wang Yong. This article is distributed under the terms of the Creative Commons Attribution 4.0 International License, which permits unrestricted use and redistribution provided that the original author and source are credited.

\author{
Wang Yong* and Jiang Xin \\ Hangzhou Robam Appliances CO., LTD. Hangzhou, China
}

\section{Abstract}

For the past few years, the principles of Chinese cuisine have triggered widespread concern. This paper reviews the principle, model, characteristics, influencing factors, and pro-spects of the heat and mass transfer of Chinese cuisine, which could provide theoretical guidance and application support for the development of Chinese cuisine.

Keywords: Chinese cuisine; Mass and heat transfer; Model; Application

\section{Introduction}

For the past few years, the principle, model, characteristics and influencing factors of Chinese cuisine have triggered widespread concern. The main heat transfer direction in cooking process is from heat source, cooking pot, liquid medium, and to food surface and center. Heat transfer can be divided into three basic ways: heat conduction, heat convection and thermal radiation [1]. Among them, container-liquid heat convection, liquid-food heat convection and food internal heat conduction are the leading process, has great influence on cooking. The law of heat transfer mainly refers to the relationship between the heat transferred in per unit time and the corresponding temperature difference in the object. The first-level formula that reflects this law is called the heat transfer rate equation. The main mass transfer direction is oil and water transferring from outside to inside or otherwise. When surface temperature of food particles exceeds the boiling point of water, evaporation will take lots of latent heat away [2].

Although there have been many automatic cooking technical solutions, there is currently no method for converting manual cooking operations into automatic cooking operations based on scientific principles, which has become an urgent problem in the current development of Chinese cooking automation. This paper reviews the principle, model, characteristics, influencing factors, and prospects of the heat and mass transfer of Chinese cuisine, which could provide theoretical guidance and application support for the development of Chinese cuisine.

\section{Principle and Model Application of Heat and Mass Transfer}

\section{Principle of heat and mass transfer}

Heat conduction is heat transfer caused by the thermal motion of microscopic particles such as molecules, atoms or free electrons when no displacement occurs among objects, which is a kind of diffusion behavior that is based on microscopic particles' random motion of the constituent materials [3]. Gases, liquids and solids will have some thermal conductivity when temperature difference exists. But, heat conduction relies on close contact, which only occurs when objects are in direct contact, and its law can be described by the Fourier definition [4].

Heat convection is heat transfer caused by the macroscopic flowing and the mixing between cold fluids and hot fluids when there exists temperature difference [5]. Apparently convective heat transfer happens/exists inside every part of the fluids. When fluids flow in a macroscopic manner, the molecules of fluids are undergoing irregular thermal motion, which means that heat convection is inevitably accompanied by heat conduction. In other words, heat convection is the combined effect of heat conduction and heat convection. When there exists temperature difference or uneven temperature distribution inside the fluids, heat convection acts based on the quantitative relationship specified by Fourier's Law [4]. 
The mechanism of heat convection is related to the heat transfer, which occurs on the film layer that adjacent to the fluids' wall and closely related with specific heat transfer process. According to the causations of the flow, heat convection is divided into two categories: natural convection and forced convection. Natural convection is caused by density change in fluids when the fluids are heated or cooled, such as oil flow in the power transformer. Forced convection is caused by external driving forces such as pumps or fans, for example, the flow of cooling water in the oil cooler or condenser is driven by pumps [6].

Whatever gas or liquids, single-phase heat convection exists when phase doesn't change. If the phase changes when materials heated or cooled, like the fluids transferring to gases or the gases transferring to fluids, between the adjacent wall of fluids and solids exists latent heat, which were respectively called boiling and condensation. Heat transfer like boiling and condensation often occur in engineering such as coal-fired power generation, nuclear power, air-condition refrigeration. The basis calculation formula of heat convection is Newtonian cooling formula [7].

Thermal radiation is a phenomenon where object possess temperature meanwhile radiates electromagnetic waves [8]. All objects with temperatures above Absolute Zero can generate thermal radiation, and the higher the temperature, the more the total radiated energy and the short-wave is. The spectrum of thermal radiation is a continuum, and the wavelength coverage can theoretically range from 0 to $\infty$. The general heat radiation mainly depends on the visible light with long wavelength and infrared rays. Since the propagation of electromagnetic waves does not require any medium, heat radiation is the only way to transfer heat in vacuum environment.

While the material emits radiant energy outward, it continuously absorbs the radiant energy emitted by surrounding objects and retransforms it into heat energy. This kind of heat transfer through objects' radiant energy emission and absorption called heat radiation [9]. If the radiation heat transfer occurs between two objects with different temperatures, the direction of the heat transfer is from the high temperature object to the low temperature one. If the two objects have the same temperature, the radiation heat transfer between the objects is zero, but the process of emission and absorption is still running, in a state of thermal dynamic balance. Both theoretical derivations and experiments show that the ability of an object to generate heat radiation is related to its thermodynamic temperature and surface properties. There is an idealized model called absolute black body, or black body for short, which is of great significance in studying problems of heat radiation.

\section{Traditional Chinese cuisine}

The complicated operation of Chinese cooking can be divided into three categories: material compatibility, cutting and heating control, which respectively correspond to the three technical elements of cooking: seasoning, knife and heating. Seasoning is a subjective technology, depending on dietary traditions and cooking techniques, isn't the key to process control. Cutting, on the one hand, gives the dish a suitable shape and taste. On the other hand, it determines the heat transfer characteristic, size. Cutting is another embodiment of heating control, as well as an important factor in the cooking process. Heating control, also called "huo-hou", is the key operation in cooking, methods like Boiling, stewing, blasting, frying, simmering, rinsing, braising, roasting, sticking, grilling are concerning heating medium, heat transfer method and heat exchange strength control [10].

The cutting and heating control determine the material temperature in cooking, which determines the chemical and physical reactions, causing varieties in cooking quality. The variety of temperature can be analyzed by process transfer principles, which includes heat transfer, mass transfer, and momentum transfer. The quality changes of cooking are analyzed by food chemistry, food physics and food microbiology, among which food chemistry and physics are key factors. Dynamics links the temperature change and cooking quality, which is the bond between the cooking methods and the cooking process [11], including the chemical and physical kinetics changes and the microbial lethal dynamics in materials.

Food quality loss kinetics in food engineering has been studied for many scholars. Food quality change generally refers to chemical, physical and microbial changes in cooking process. Among them, chemical reaction kinetics is the basic theoretical model, which can reflect those changes properly and have been widely used. For example, the changes of heat sensitive materials such as vitamins, pigments or enzymes, are obligated to the first-order reaction kinetics during the heat treatment in most case [12].

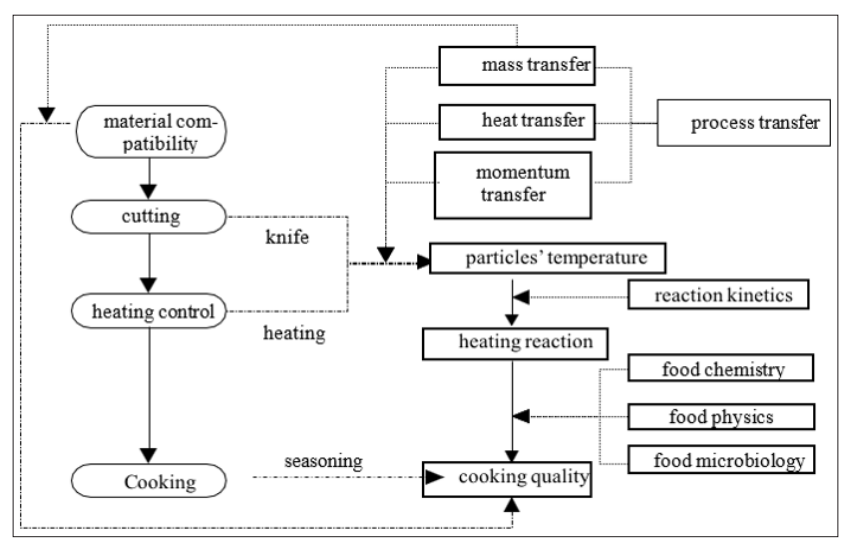

Figure 1: Relationship between the various disciplines of cooking. 
In cooking process, the study of kinetics is based on the Bigelow model (D-Z model) of food science [13], also can be expressed by the general chemical reaction kinetics Arrhenius model ( $\kappa$-Ea model). Usually, the Arrhenius model is applied to reflect the quality change such as storage, heating, concentration, etc. While $\mathrm{Z}$ model is often used in sterilization operation. The two models are common in food heat treatment, which have the same theoretical significance, and whose calculation results are similar too, so sometimes they can replace each other [14]. In the food industry, the first-order reaction kinetic models have widespread uses, such as calculating the loss of vitamins, enzymes, color heat loss and microbial death. Therefore, process transfer-reaction dynamic-food quality change determines the quality of cooking, also is the core principles of cooking. Figure 1 shows the relationship among cooking process, internal changes and related subjects.

\section{Model application of heat and mass transfer}

Heat transfer exists everywhere, so it is widely used in various fields of science technology and engineering application which not only involves traditional industries such as energy power, chemical industry, metallurgy, transportation and mechanical engineering, but also widely ap-plied to many high-tech fields such as aerospace, microelectronics, nuclear energy, new energy, biomedical science and agricultural engineering. The application of heat transfer in science technology and engineering can be divided into three types: enhanced heat transfer, weakened heat transfer (thermal insulation) and temperature control [15].

\section{Enhanced heat transfer}

Enhanced heat transfer is to increase the transfer of heat in certain conditions, in order to im-prove the utilization of equipment, save energy or meet special process requirements. The enhanced heat transfer technology uses various forms of heat exchanger such as finned tubes, porous surface tubes, surface roughening tubes, tube inserts to add electric fields, magnetic fields, ultrasonic waves and other auxiliary devices, which aim to facilitate the medium in the flow of heat exchange devices to generate turbulence, thin the boundary thermal resistance, and strengthen the role of the heat transfer surface, thereby achieving the purpose of effectively transferring heat [16]. The application of enhanced heat transfer technology in heat ex-changers mainly meets the heat requirements in the most economical (small-size, small-mass, low-cost) equipment, or effectively cools the hightemperature components so as to make them run under safe and reliable working conditions.

\section{Weaken heat transfer}

Weaken heat transfer is used to reduce heat loss occurring in high-temperature equipment and reduce the loss of cooling in low-temperature equipment [17]. For example, taking measures to a cryogenic vessel which holds liquid nitrogen and liquid oxygen can reduce heat transfer in the direction perpendicular to the wall of the Dewar to one thousandth of that before taking measures, or even less, thereby effectively preventing the evaporation of low temperature liquids in the bottle and reducing the loss of cooling.

\section{Temperature control}

The problems in temperature control are mainly reflected in the cooling of electronic devices, the protection of spacecraft and other aspects. According to statistics, the main cause of current electronic device damage is thermal damage, which means that the operating temperature exceeds the allowable value, which leads "how to control the temperature of the highly integrated chip" be-comes more and more urgent [7]. Heat transfer has formed a micron-nano research direction to solve the problems of heat transfer and flow in micron-nano size. Experts and scholars have developed new temperature control methods, including phase change temperature control technology, jet impact enhancement enhanced heat transfer technology, liquid metal heat dissipation technology, solid-state radio frequency heating technology, heat pipe technology, electrostatic cooling technology, etc.

\section{Heat and Mass Transfer in Traditional Chinese Cuisine}

Li Deng [2] believed that typical Chinese cuisine was the process of heat and mass transfer, relative motion and quality change occurred between food and water in open containers, which meant that process transfer, reaction kinetics and quality change was the core principle of Chinese cuisine.

\section{Steaming}

Tang Shuai [18] found that the heat conduction performance of filling increased with heating time increased, while the dough decreased. Steaming power and heating time had different influences on heating rate of Stuffed Bun on different floors. Under different steaming power, the maturity process of Stuffed Bun was divided into three stages, which was derived by established kinetic mathematical model of the steaming characteristic indexes (rate of steaming, starch gelatinization, and soluble protein denaturation). Results showed that the indexes suffered decreased rapidly and then slowly as the heating time increased. Meanwhile, there was a significant correlation among dough temperature, the RVA spectrum and steaming property indexes through correlation analysis. And Stuffed Bun's mature time could be predicted by establishing temperature model of dough and filling.

Li R [19] found that the maturity process of Stuffed Bun could be divided into three stages according to its central heating rates (low, high and steady, respectively). In the first two stages, the central heating rate is positively correlated with steam volume. The fitting equation of central heating rate and steam volume was $y=0.0003 x+0.1158, R 2=0.96$. The fitting equation of pressure difference (between inside and outside of Stuffed $\mathrm{Bu}$ ) and steam volume was $\mathrm{y}=0.0283 \mathrm{x}+5.1067, \mathrm{R} 2=0.97$. And the fitting equation of pressure difference and central heating rate was $y=0.0152 x+0.0597, R 2=0.998$. Therefore, the central heating rate and pressure difference of Stuffed Bun could be estimated according to steam volume, thereby providing technical guidance for industrial production.

During the steaming process of Stuffed Bun, with time prolonged, the pressure difference be-tween outside and inside 
of Stuffed Bun rose first and then decreased, which decreased faster along with steam volume being larger. At steaming time of 1 min, when the pressure difference reached 0.14 bar, it made good cracking effects on top of Stuffed Bun. At steaming time of 3 min, when the pressure difference reached 0.14 bar, the juice from filling would expose from the bottom of Stuffed Bun, making the quality of Stuffed Bun low [19].

During steaming process of Stuffed Bun, the moisture of the dough increased first and then tended to be steady, while the filling decreased at different degrees. The rate of moisture content in dough and filling increased quicker at high steam volume $(200 \mathrm{~mL}$, $150 \mathrm{~mL}, 100 \mathrm{~mL})$, than that at low steam volume $(75 \mathrm{~mL}$ and $50 \mathrm{~mL})$. According to steaming kinetic model of Stuffed Bun, the steaming rate decreased quickly first and then slowly with time prolonged at different steam volumes. In the first stage (first $2 \mathrm{~min}$ ), the steaming rate constant was positively related with steam volume when steam volume was less than $100 \mathrm{~mL}$, while it changed opposite when steam volume exceeded $100 \mathrm{~mL}$. In the second stage ( $2 \sim 5 \mathrm{~min}$ ), the steaming rate constant was positively related with steam volume. In the third stage (over $5 \mathrm{~min}$ ), the steaming rate constant at high steam volume was lower than that at low steam volume. The gelatinization rate of Stuffed Bun was consistent with the steaming rate. As steam volume increased, the hardness of Stuffed Bun generally decreased, and the elasticity slowly increased correspondingly. Statistics showed that Stuffed Bun cooked at steam volume of $100 \mathrm{~mL}$ had the lowest adhesion, and there was no significant difference in cohesiveness at steam volume of $75 \mathrm{~mL}$, but significantly higher than at steam volume of $50 \mathrm{~mL}$ [19].

\section{Quick-frying}

Quick-frying is one of representative cooking methods in traditional Chinese cuisine, by which heat transfer and mass transfer in food particles occur simultaneously, involving coupling of temperature, concentration and pressure. It has remarkable unsteady state so that it is hard to make comprehensive analysis in traditional method. But, the numerical simulation technology can effectively solve this problem [20]. Based on the theory of porous media and the principle of unsteady heat transfer, the mathematical model of heat and mass transfer during the process of quick-frying was established [21]. Meanwhile, comprehensive simulation and verification experiment for temperature and moisture during quick-frying were carried out. The result showed that with data accumulated, heat and mass transfer process during quick-frying can be analyzed and predicted by adjusting the corresponding value [22].

Oil frying, one of typical quick-frying, using oil as the heattransfer medium, had properties of intense and remarkable unsteady process. Based on energy balance and heat and mass transfer in oil frying process, the control equation of heat transfer and endothermic power was established. And a method for measuring the endothermic power of food system in oil frying process was verified by experiment. Stirring frequency in oil frying process and the distance from the pot bottom to heat source are influencing factors to food system's endothermic power. The result showed that the distance from the pot bottom to the fire source, endothermic power was in linear negative relation when stirring frequency was identical. When the distance was consistent, endothermic power increased linearly with stirring frequency $[21,23]$.

Huang D [24] used Time-Temperature Integrators (TTIs) to simulate temperature time change of target quality parameters, and evaluated the heat treatment effect, respectively. It was mainly focused on protein materials such as meat and viscera cooked by quick-frying. The $\mathrm{Z}$ value of protein denaturation was $5 \sim 10{ }^{\circ} \mathrm{C}$, and it took $\alpha$-amylase with $\mathrm{Z}$ value of $7.35{ }^{\circ} \mathrm{C}$ as TTIs indicator. By measuring the change before and after heat treatment of the TTIs indicator, along with the mathematical model of $\mathrm{Z}$ value, the heat and quality dynamics was estimated.

Li WX [14] studied kinetic model of quality change using oil as heat transfer medium and pork tenderloin and spinach as food materials. And terminal maturity values of different food materials were determined. It showed that there was significant correlation between terminal maturity value and the component of raw food materials.

Zhou J et al. [25] developed a data system based on Deng L et al. [2], which could realize multi-channel automatic acquisition of cooking temperature and automatic integral calculation of $\mathrm{M} / \mathrm{O}$ value. However, reaction sequences and kinetic parameters such as $\mathrm{k}$ value and $\mathrm{D}$ value could not be determined by the kinetic experiment, so that food maturity evaluation was just depended on subjective parameters from sensory evaluation [6]. Therefore, redefined food maturity combined with the sensory evaluation was equivalent heating time to reach a certain temperature, when foods reach a certain quality estimated by sensory evaluation of certain select persons.

\section{Baking}

Zhou H [26] made fresh chicken wings first wet-marinated and secondly dried with hot air $\left(60 \sim 100{ }^{\circ} \mathrm{C}\right)$ until the moisture was reduced to $45 \%$. Thirdly, the chicken wings were baked at $175{ }^{\circ} \mathrm{C}$ until the moisture was reduced to $30 \%$. Finally, the finished product came out through sterilization and vacuum packaging.

Results showed that the baking of chicken wings was slowdrying process of internal water diffusion, and the moisture and drying rate were significantly affected by hot air temperature $(\mathrm{P}<0.05)$. The drying rate and Deff(diffusion activation energy) of chicken wings were increased together with the hotair temperature, about $3.09 \sim 11.13 \times 10(-9) \mathrm{m}^{2} / \mathrm{s}$ and $12.14 \sim 33.08 \mathrm{~kJ} / \mathrm{mol}$ at $60 \sim 100$ ${ }^{\circ} \mathrm{C}$, respectively. And further studies showed that Page equation was the fit drying model $(\mathrm{R} 2=0.9994, \chi 2=0.000041)$, which could accurately predict the moisture of chicken wings during the whole baking process.

\section{Characteristics}

Especially in the quick-frying process, heat transfer was instable when food particles could not completely immerse in the liquid at relatively small liquid-solid ratio, even during the stirring. 
On the one hand, the surface areas of container-liquid and the food particles were unstable during the stirring. On the other hand, the food particles were contact directly with the container or ex-posed to air, which made the heat transfer of food surface intermittent, and the heat transfer coefficient between container, liquid and particles changed. Luckily, stirring operation could reduce the instability to some extent at special liquid-solid ratio. Meantime, as long as the stirring operation was substantially stable, the heat transfer coefficient was precisely measurable and could be used for analytical calculations [6].

It is worth mentioning that, since food particles and water moved simultaneously during the stirring and the liquid phase was changed in the same way, the heat transfer coefficients of container-liquid and liquid-particles were changed close in similar value. And the contact area of container liquid and liquid-particles had big effects on heat transfer. The surface area of liquid particles was significantly larger than that of container-liquid when food particles were relatively small, thus food particles could absorb much more heat from the liquid, whose energy supplied by container. When food needed to be cooked more rapidly by the traditional Chinese cuisine, i.e. quick-frying, we could use methods like preheating cooking oil to increase the heating rate and temperature of the food particles, which meets the principles of heat transfer [2].

\section{Heat and Mass Transfer in Modern Chinese Cuisine}

\section{Vacuum frying}

Vacuum Frying (VF) has the characteristics of low fuel consumption, little influence on nutrients and good product flavor which make it have been favored by Chinese cooking in recent years. Scholars have studied heat and mass transfer theory of vacuum frying to provide theory basis for process and equipment optimization. Wang et al. [27] studied the changes of moisture and fat content of fresh mushrooms during the combined drying process, osmotic de-hydration along with vacuum frying, found that the moisture and fat content of fresh mushrooms gradually decreased with the prolongation of penetration time, as well as fat absorption rate. According to the correlation analysis of K Pearson, the fat content has a very significant correlation with its initial moisture content. Combining the first-order reaction kinetics of vacuum frying and the water diffusion law of osmotic dehydration Fick, the moisture content in the combined drying process of fresh mushrooms is obtained.

Fan L [28]; Fan [29] and Song X [30] respectively measured the convective heat transfer coefficient of vacuum fried carrot slices and potato chips using the one-dimensional heat transfer model and established the model of vacuum frying dehydration using the first-order reaction kinetics equation. Troncoso [31] and Pedreschi [17] respectively used a one-way diffusion model with a constant effective diffusion coefficient and a variable effective diffusion coefficient model proposed to describe the vacuum oil dehydration process and concluded that the effective diffusion coefficient variable model is more accurate. Moreira et al. [32] used a logistic kinetic model to describe the oil absorption law of vacuum fried potato chips, indicating that the fat content increased exponentially and eventually stabilized. Yagua et al. [33] used the convective heat transfer theory of Hubbard \& Farkas [34] to obtain the empirical equation of heat transfer in vacuum frying through sample heat and quality parameters, and determine the range of heat transfer coefficient, which increases with the increase of frying temperature. The theoretical research on heat and mass transfer of vacuum frying technology has effectively pro-moted technological reform and progress and provided new impetus for the development of high-yield, high-efficiency and high-quality vacuum frying industry.

\section{Microwave}

Microwaves generally refer to electromagnetic waves with a wavelength of $1 \mathrm{~mm}$ to $1 \mathrm{~m}$ and a frequency range of $300 \mathrm{MHz}$ to $300 \mathrm{GHz}$. The microwave frequencies commonly used in industry are $915 \mathrm{MHz}$ and $2450 \mathrm{MHz}$. Microwave has the following characteristics: heating from internal, fast and efficient, little impact on flavor and nutrients, low energy consumption, convenient operation and low maintenance cost [35]. The comparison between the method of microwave energy internal heating and conventional external heating is shown in Figure 2.

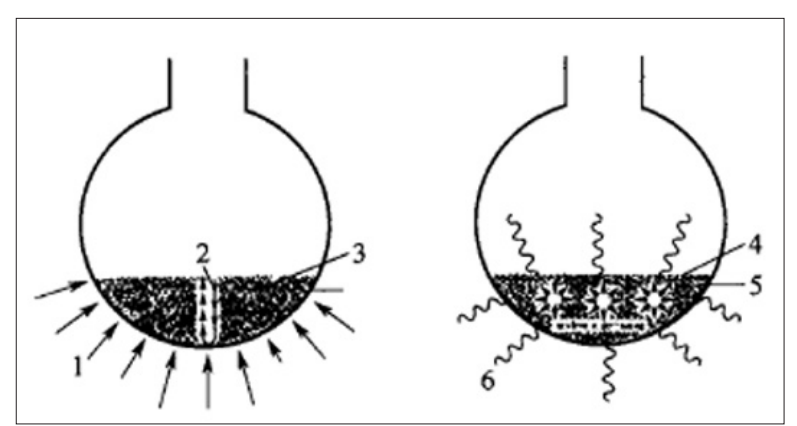

Figure 2: Traditional external heating (left) and microwave internal heating (right).

According to the researchers, the microwave absorption and temperature distribution of the ma-terial is related to its dielectric properties, location, the microwave field and intensity, which is usually calculated by Lambert's law and Maxwell's equation [7,36]. Knoerzer et al. [12] designed a three-dimensional computer model describing the temperature variation in any shape food material with time in the microwave field, which is more accurate and convenient for the simulation and prediction. The heat and mass transfer process of microwave dehydration is related to the microwave field strength and material composition, shape, loading, processing temperature and processing environment. Abbasi et al. [37] used a one-dimensional steady-state system finite difference method to establish a heat and mass transfer model of spherical porous food materials in micro-wave-assisted fluidized bed drying, and used different microwave power, air flow rate and air temperature to determine the moisture of green beans. The content change was verified to obtain the optimized heat and mass transfer coefficient.

Figiel [38] used the four indexes (water content, relative volume, compressive strength and rehydration rate) to establish the coefficient equation, and the moisture content and quality changes of vacuum-dried garlic and garlic slices were comprehensively 
described and predicted. Motavali et al. [39] compared the accuracy of the artificial vacuum network and the Madill empirical equation to establish a heat and mass transfer model of microwave vacuum dried cherry, indicating that the artificial neural network model has higher accuracy. Cui [40] based on the balance of sensible heat, latent heat and microwave energy absorption of materials, established a mathematical model of microwave vacuum drying through experimental data and verified its accuracy. Malafronte et al. [41] used a variety of physical quantities combined with dry kinetic equilibrium equations and Maxwell's equation establishes heat and mass transfer model of microwave convection drying potato block, and comprehensively describes all the characteristics of the materials in the drying process of microwave convection and provide a basis for the prediction of the microwave combined drying process.

\section{Combined cooking}

The combination of microwave and vacuum frying is also an important direction in the new Chinese cooking. Su Y [35] explored the law and mechanism of microwave vacuum frying dehydration and lipid reduction. The research indicates that the internal heating of microwave and the heat convection of fried oil are synergistic, made the heat transfer direction and water vapor migration of material tends to be consistent, the driving force for dehydration increases to achieve more efficient frying. A thin layer dehydration kinetic model of microwave vacuum deep-fried potato chips was established. Microwave ( $600 \mathrm{~W} 1000 \mathrm{~W}$ ) increased the moisture diffusion coefficient of VF potato chips by about 33.3\% 47.6\%. The first-order reaction kinetics law was used to simulate the change of fat content of MVF potato chips and established its lipo-suction model. The surface and structural fat content of MVF samples were lower than that of VF samples by quantitative determination and fat distribution and microstructure observation. The size and distribution of cell morphology and porous structure determine the fat content of vacuum fried products. Microwaves increase the vapor pressure of VF potato chips, accelerate the formation of dry porous structures, reduce the adhesion and penetration of fat in porous structures, while maintaining the open cell morphology and producing more tiny porous structures, making More surface fat is removed by centrifugation and reduce the fat inside the inhaled structure, thus reducing the surface fat and structural fat content of the potato chips, revealing the microwave vacuum frying and lipidlowering mechanism [32].

\section{Characteristics}

A. Microwave has the advantages of high heating efficiency, scarcely change in flavor and nutrients, low energy loss, convenient operation and low maintenance cost, which is widely used in food processing such as dehydration, concentration, sterilization, thawing and baking. The microwave dehydration technology utilizes the characteristics of internal heating, make the material heat transfer and water diffusion direction are consistent, improve the driving force of heat and mass transfer and drying efficiency.
B. Vacuum frying, a drying method accompanied by heat and mass transfer, has the following advantages:

a) low temperature and less nutrient components loss;

b) rapid evaporation of water by decompression;

c) aroma and flavor retention;

d) has puffing effect and rehydration quality;

e) less fuel consumption and slow cracking rate of oil [22]. During the frying process, heat is transferred from frying medium to material surface by heat convection and then transferred to material center by heat conduction. With the continuation of frying process, the water evaporation interface gradually migrates to the center, the surface fragile shell begins to form, and gradually migrates to the center, and the fat is gradually absorbed by the material. As the frying time extended, the diameter, thickness, and basic characteristics such as center temperature, moisture and fat content, and drying rate will change significantly.

C. Microwave synergistic vacuum frying can improve the processing efficiency of food. Com-pared with vacuum frying, it reduces frying time and fat content, and the color and texture of the product can be significantly improved. Microwaves maintain the cell morphology of the sample surface and produce lots of tiny porous structures. Microwave synergistic vacuum frying is a slow-down drying process which conforms to the first-order dehydration kinetics. Its dehydration rate increases significantly with the increase of microwave power, frying temperature and vacuum, and the fat content and crushing force are significantly reduced. The color difference increases with the increase of frying temperature and microwave power, while decreases as the degree of vacuum increases. The increase of slice thickness reduces the dehydration rate and fat content of microwave synergistic vacuum frying, increasing the loading factor within a certain range is beneficial to improve the microwave utilization rate; the three-dimensional movement of liquid medium and material makes the microwave synergistic vacuum frying have better drying uniformity [35].

\section{Factors of Heat and Mass Transfer in Chinese Cuisine}

As we know, the purpose of cooking is to mature food ingredients and achieve the best edible quality. Maturation changes include protein denaturation, starch gelatinization, pectin hydrolysis, water swelling of cellulose, and the formation of color and flavor. Most articles believe the three key factors, seasoning, cutting and heat, constitute cooking technology, of which heat is the key point. They considered heat as a comprehensive control of effective power material movement and heating time to maintain cuisine maturity and best quality, where operations that will influence heat convection like cutting isn't concluded [24].

\section{Suitable size of food}

Food shaping is the technology of preparing food to specific shape for cooking, including cutting and forming, which cuts food 
into pieces, slices, strips, granules and so on, or forms food into a mixture like cake, jelly, or cookies. The characteristic size is directly related to the quality. For example, the volume is reduced after meat being cut, so that the temperature and the time re-quired to reach maturity and the quality loss rate can be greatly reduced [5].

\section{Temperature}

Maturity is the result of temperature and time accumulation in cooking, where temperature plays a decisive role. In cooking research, solid food is a main part, in which the heat transfer process is unstable, and temperature is the key factor. For instance, the maturity of meat is directly related to temperature distribution, which determined by heat exchange on the surface between water and meat and the internal heat convection, obeying the principle of unstable heat transfer [9]. All the factors affecting the temperature of the cooking materials are the factors affecting cooking heat transfer, such as kinetic parameters, heating equipment parameters, parameters of the heat transfer liquid medium, particle parameters, environmental parameters, fluid parameters in the particle pores, convection transfer coefficient and related operation parameters [2].

\section{Pot}

The main purpose of making the pot is to obtain rapid heating and uniform temperature distribution of the bottom, thereby allow the food particles heated evenly and quickly. However, during the cooking process, the bottom of the pot is heated by the non-steady state, which makes it difficult to obtain a uniform temperature on the wall where in contact with the food [15]. It is found that the material of pots will produce thermal resistance. The stainless steel with higher thermal resistance has slower temperature rise and uneven temperature distribution, while the copper and aluminum with lower thermal resistance have faster temperature rise and uniform temperature distribution, which means the thermal resistance coefficient of the pot can be set as a key parameter reflecting the heat transfer characteristics of the pot.

When the cooking preheating oil temperature is higher or the stirring frequency is lower, the smaller the thermal resistance coefficient of pot has, the faster the food quality change rate is, and at the same time, it is more likely to have dishes burnt, and the cooking operation is difficult. While the larger the thermal resistance coefficient of pot is, the longer the time for food maturing, the scorching time and the interval time are, the easier the cooking operation is, which is beneficial for non-professionals to use [21].

\section{Process enhancement and buffering}

Process enhancement and buffering like stirring in "fried", the stirring and pressing in "stir", increase heat convective coefficient or contact area of heat conduction, also enhance the heat or mass transfer process. The "Guahu”, "Shangjiang", "Gouqian" means that the paste formed by means that the paste formed by adding edible hydrocolloids is attached to the surface of solid food, slowing down the heat transfer process, and buffering the heat transfer process. For example, during the frying process, "Guahu" changes the heat medium from oil to water and steam. The use of water and oil heat transfer in Chinese cooking can reduce surface overheating caused by direct conduction between cookware and food, so that improving the quality of food [14]. "Guahu", "Shangjiang" and "Gouqian" are widely used in Chinese cooking, acting as process strengthening or buffering. They are complex and important. Their other functions like forming a high concentration layer on the surface of the food to enhance mass transfer, participating in the food chemical reaction to optimize texture, forming a taste improver by itself, forming a color and acting as a color improver.

\section{Prospects}

Chinese cooking is one of the cores of traditional Chinese food technology and has an important impact on the food industry and the development of the national economy. With the rapid growth of the national economy and the improvement of people's living standards, Chinese cooking ur-gently need and will gradually realize industrialization and modernization and will have a huge impetus to the development of social economy. According to the model of mass and heat transfer of Chinese cooking, the system and theoretical basis are established, and its development to engineering and standardization is promoted, thereby realizing its large-scale continuous, industrialized production, standardization, mechanization and automation can be fulfilled [42].

In addition, based on the model of mass and heat transfer in Chinese cooking, the microcomputer can be used to control the cooking program, and the digital programming can solve the problem of cooking program. Electromechanical structural design is adopted to bring power and skill, and finally the heat source can be converted by using clean natural gas or utilizing, and a cooking technology can be developed consisting of three aspects: an intelligent control system, a functional realization structure and an interactive system. Many cooking devices are connected in the Internet mode, and the cooking, the taste, and the dishes can be adjusted on the mobile terminal, so that people no longer have to worry about upgrading their cooking techniques. The development of cooking technology in the Internet will develop in the direction of intelligence, humanization and remoteness which makes Chinese cooking technology preserved, and sustainable, stepping from the traditional kitchen to the stage of the world [43].

\section{References}

1. Zhu S, Liu Y, Lin Z (2011) Engineering thermodynamics. ( $2^{\text {nd }}$ edn), Tsinghua University Press, China.

2. Deng L (2013) Construction of mathematical model of heat and mass transfer process in Chinese cooking. Transactions of the Chinese Society of Agricultural Engineering 29(03): 285-292.

3. Chen X (1996) Kinetic theory and its application in heat transfer and flow research Beijing: Journal of Tsinghua University Science and Technology, China.

4. Datta AK (2007) Porous media approaches to studying simultaneous heat and mass transfer in food processes. I: Problem formulations. Journal of Food Engineering 80(1): 80-95.

5. Tao W, Yang S (2006) Heat transfer. Higher Eduction Press, Beijing, China.

6. Li J, Deng L, Jin Z, Yan Y (2017) Modelling the cooking doneness via integrating sensory evaluation and kinetics. Food Research International 92: 1-8. 
7. González CS, González MF, Malo AL, Morales MES (2012) Recent studies related to microwave processing of fluid foods. Food and Bioprocess Technology 5(1): 31-46.

8. Jiang S, Zhanghong L (2018) Effect of thermal radiation on the nominal thermal conductivity of carbon nanotube sheet. Materials Reports 32(S2): 565-567.

9. Yuan Y, Zhu X (2018) Influence of thermal radiation on thermal simulation about electronic equipment. Machinery 45(1): 34-36.

10. Deng L (2013) Kinetic functions, optimizing model and definition of "Huohou" for Chinese cooking. Transactions of the Chinese Society of Agricultural Engineering 4: 278-284.

11. Deng L, Zhengyu J (2006) Analysis and research system of Chinese cooking process. Principle Food \& Machinery 22(6): 140-143.

12. Knoerzer K, Regier M, Schubert H (2008) A computational model for calculating temperature distributions in microwave food applications. Innovative Food Science \& Emerging Technologies 9(3): 374-384.

13. Tian W, Xu R (2000) The relationship of the Arrhenius model and Z-value model. Journal of Tianjin University of Light Industry 4: 1-6.

14. Li W (2015) Study on the kinetics of meat and vegetables in cuisine based on the theory of maturity value. Guizhou University, China.

15. He Y, Zhang X, Meng X (2015) Heat transfer. Chemical Industry Press, Beijing, China.

16. Wang Y (2011) Study on the effects of lotus-seed-paste preparing and baking process influencing the qualities of Cantonese lotus-seed-paste moon cakes. Journal of South China University of Technology, China.

17. Pedreschi F, Hernández P, Figueroa C, Moyano P (2005) Modeling water loss during frying of potato slices. International Journal of Food Properties 8(2): 289-299.

18. Tang S (2017) Study on the process of the thermal history and its effect on the edible quality of baozi. Journal of Zhejiang Gongshang University, China.

19. Li R (2015) Research on the quality of the composite leavening and steaming of the natural fermented dough of Cantonese barbecued pork bun. Journal of South China University of Technology, China.

20. Deng L (2013) Numerical simulation, optimization, study of technical features and operational parameters for Chinese Cuisine. Transactions of the Chinese Society of Agricultural Engineering 29(5): 282-292.

21. Cui J (2017) Simulation by CFD and energy consumption analysis for stir-frying with oil. Guizhou University, China.

22. Peng J (2018) Evaluation method and application optimization of Chinese wok based on maturity value theory. Guizhou University, China.

23. Cui J, Deng L, Wang X, Li SY, Wang L (2017) Study on endothermic power of food system in oil frying. Food Industry Technology 38(7): 182-187.

24. Huang D (2016) The establishment and study on the application of cuisine TTIs. Guizhou University, China.

25. Zhou J, Deng L, Yan Y, Li H (2013) Development of system on data acquisition and analysis of heat transfer and kinetics for Chinese cuisine. Transactions of the Chinese Society of Agricultural Engineering 29(23): 241-246.
26. Zhou H (2014) Research on the heat and mass transfer and kinetics of retort pouch packed roast broiler chicken wings during processing. Journal of South China University of Technology, China.

27. Wang X, Shujing X, Li L, Yang D, Guan J, et al. (2015) A Kinetics Analysis of the Combined Drying of the Osmotic Dehydration and Vacuum Frying for the Fresh mushrooms. Hubei Agricultural Sciences 54(21): 5368-5371.

28. Fan L (2005) Study on processing and storage of low-fat carrot chips. Jiangnan University, China.

29. Fan L, Han Z (2012) Study on the drying characteristics and products quality changes during vacuum frying. Science and Technology of Food Industry 33(8): 194-197.

30. Song X (2008) Study on the process of low oil content potato chips. Jiangnan University, China.

31. Troncoso E, Pedreschi F (2009) Modeling water loss and oil uptake during vacuum frying of pre-treated potato slices. Food Science and Technology 42(6): 1164-1173.

32. Moreira RG, Silva PF, Gomes C (2009) The effect of a de-oiling mechanism on the production of high-quality vacuum fried potato chips. Journal of Food Engineering 92(3): 297-304.

33. Yagua CV, Moreira RG (2011) Physical and thermal properties of potato chips during vacuum frying. Journal of Food Engineering 104(2): 272 283.

34. Hubbard LJ, Farkas BE (1999) A method for determining the convective heat transfer coefficient during immersion frying. Journal of Food Process Engineering 22(3): 201-214.

35. Su Y (2018) Studies on the effects and mechanisms of microwave and its synergistic treatments on the efficiency and quality of vacuum fried potato chips. Jiangnan University, China.

36. Chandrasekaran S, Ramanathan S, Basak T (2013) Microwave food processing-A review. Food Research International 52(1): 243-261.

37. Souraki BA, Mowla D (2008) Simulation of drying behavior of a small spherical foodstuff in a microwave assisted fluidized bed of inert particles. Food Research International 41(3): 255-265.

38. Figiel A (2009) Drying kinetics and quality of vacuum-microwave dehydrated garlic cloves and slices. Journal of Food Engineering 94(1): 98-104.

39. Motavali A, Najafi GH, Abbasi S, Minaei S, Ghaderi A (2013) Microwavevacuum drying of sour cherry: comparison of mathematical models and artificial neural networks. J Food Sci Technol 50(4): 714-722.

40. Cui Z (2004) Mathematical modeling of microwave-vacuum drying and its application in food processing. Jiangnan University, China.

41. Malafronte L, Lamberti G, Barba AA, Raaholt B, Holtz E, et al. (2012) Combined convective and microwave assisted drying: Experiments and modeling. Journal of Food Engineering 112(4): 304-312.

42. Wu D (2016) On the present situation and application of Chinese cooking. Modern Food Science and Technology (3): 3-4.

43. Yu D, Deng X (2016) Research on sustainable design of Chinese intelligent cooking machine under the internet. Sciences and Wealth (6): 712-713.

For possible submissions Click below: 